\title{
Aprendizaje activo mediante juego de roles en Ingeniería Biomédica: negociando la adquisición de un sistema de información hospitalaria
}

\section{Carlos Sáez $^{\text {a,b }}$, Elies Fuster-García ${ }^{c}$, Pablo Ferri ${ }^{\text {a,b }}$ y Juan M García-Gómez ${ }^{\text {a,b }}$}

${ }^{a}$ Biomedical Data Science lab, Instituto Universitario de Tecnologías de la Información y Comunicaciones, Universitat Politècnica de València, España (*corresponding author: carsaesi@upv.es), ${ }^{b}$ Departamento de Física Aplicada, Universitat Politècnica de València, España, ${ }^{\mathrm{C} D e p a r t m e n t ~ o f ~ D i a g n o s t i c ~ P h y s i c s, ~ O s l o ~ U n i v e r s i t y ~ H o s p i t a l, ~}$ Oslo, Norway

\section{Abstract}

The Health Information Systems and Telemedicine I course of the Degree in Biomedical Engineering of the Universitat Politècnica de València, Spain, offers students competences in the field of computer science applied to medicine. Among its learning outcomes is the design and management of a hospital information system. These learning outcomes require the definition and integration of multiple software components specialized in different medical and management domains, with a complex interaction between them, and with variability associated with the characteristics of each hospital. Active learning through role-playing is a learning strategy that is well suited for learning these types of competences. We present a new proposal for active learning through role-playing in which students, representing heads of the different services of a hospital and software companies, negotiate the acquisition of a new component of information systems according to the needs of their department and global needs to the hospital, internalizing the design of components and their interactions, while working transversal competences such as teamwork and leadership and effective communication, among others. Additionally, towards a continuous improvement, after the session we collect through voluntary on-line questionnaires the students' opinions about the activity and about the perceived learning. Applied since the 2018-19 academic year, the activity has demonstrated its effectiveness, accomplishing the pedagogical objectives of the subject, with a good appreciation of the learning received by the students, and presenting a high rate of feedback.

Keywords: role-playing, active learning, biomedical engineering, medical informatics, medicine

\section{Resumen}

La asignatura Sistemas de Información y Telemedicina I del Grado en Ingeniería Biomédica de la Universitat Politècnica de València ofrece a los alumnos competencias en el ámbito de la informática aplicada a la medicina. Entre sus resultados de aprendizaje encontramos el diseñar y gestionar un sistema de información hospitalaria. Estos resultados requieren definir e integrar múltiples componentes software especializados en distintos dominios médicos, con una compleja interacción entre ellos, y con variabilidad asociada a las características de cada hospital. Presentamos una nueva propuesta de aprendizaje activo mediante juego de roles en la que los alumnos, representando a jefes de distintos servicios de un hospital y compañias de software, negocian la adquisición de un nuevo componente de sistemas de información en función de las necesidades de su departamento y globales al hospital, interiorizando el diseño del mismo e interacciones entre componentes, y trabajando competencias transversales como trabajo en equipo y liderazgo y comunicación efectiva. Hacia una mejora continua, recogemos mediante 
cuestionarios on-line retroalimentación de los alumnos sobre la actividad. Aplicada desde el curso 2018-19, la actividad ha demostrado su efectividad, cumpliendo los resultados de aprendizaje, con una buena apreciación del aprendizaje recibido por los alumnos, y una elevada tasa de retroalimentación.

Palabras clave: juego de roles, aprendizaje activo, ingeniería biomédica, informática, medicina.

\section{Introducción}

Las estrategias de aprendizaje activo ayudan a que los alumnos se involucren como participantes activos en el proceso de aprendizaje-enseñanza, permitiendo una mayor proximidad a los resultados de aprendizaje y competencias transversales esperadas que los métodos pasivos tradicionales. Entre las distintas estrategias de aprendizaje activo encontramos el juego de roles, mediante el cual los estudiantes aprenden a través de la simulación de experiencias reales mediante la representación de determinados roles asociados a las competencias a desarrollar durante su futuro profesional. El juego de roles, sirve a su vez de estrategia para la evaluación de dicho aprendizaje (Gaete-Quezada, 2011).

La asignatura Sistemas de Información y Telemedicina I del Grado en Ingeniería Biomédica de la Universitat Politècnica de València ofrece a los alumnos competencias en el ámbito de la informática aplicada a la medicina. Entre sus resultados de aprendizaje se encuentra el diseñar y gestionar un sistema de información hospitalaria, soporte informático a las actividades de salud y gestión desarolladas en todos los servicios de un hospital. Estos resultados requieren, en primer lugar, definir, diseñar e integrar múltiples componentes software especializados en distintos dominios médicos y de gestión, con una compleja interacción entre ellos, y con variabilidad asociada a las características de cada hospital. Y en segundo lugar, dada la interdisciplinaridad de la disciplina, es necesario describir conceptos biomédicos de las distintas especialidades clínicas y de gestión clínica involucradas.

El juego de roles es una estrategia de aprendizaje bien establecida en el ámbito de las ciencias de la salud desde hace décadas (Simpson, 1985; Steinert, 1993; Joyner and Young, 2006). En el ámbito de la ingeniería, recientemente también se ha demostrado la efectividad y la mejora en el aprendizaje mediante el uso del juego de roles como estrategia de aprendizaje (Ponsa et al., 2010; Svensson and Regnell, 2017; McConville et al., 2017). Por lo tanto, el juego de roles se postula como una potencial estrategia de aprendizaje activo para facilitar el aprendizaje de los sistemas de información hospitalaria en ingeniería biomédica dada su diversidad, multidisciplinaridad y complejidad anteriormente descritas.

En este trabajo presentamos una nueva propuesta de aprendizaje activo mediante juego de roles aplicada desde el curso 2018-19 en la que los alumnos de ingeniería biomédica, representando a jefes de los distintos servicios de un hospital y compañías de software, negocian la adquisición de un nuevo componente de sistemas de información en función de las necesidades de su departamento y globales al hospital, interiorizando el diseño del mismo e interacciones entre componentes, al mismo tiempo que trabajando competencias transversales como trabajo en equipo y liderazgo y comunicación efectiva, entre otras.

\section{Objetivos}

Los objetivos de este trabajo son: (1) diseñar una innovación docente basada en juego de roles para el aprendizaje de resultados y adquisición de competencias transversales en sistemas de información 
hospitalaria en ingeniería biomédica, (2) diseñar una rúbrica de evaluación para dicha actividad de aprendizaje y (3) evaluar el aprendizaje percibido por los alumnos mediante esta estrategia.

Esta propuesa se enmarca en la asignatura Sistemas de Información y Telemedicina I del Grado en Ingeniería Biomédica de la Universitat Politècnica de València. De las 5 unidades temáticas de la asignatura (UD1. Organización de sistemas de información de salud, UD2. Historia Clínica Electrónica y estándares de HCE, UD3. Herramientas para el soporte a la decisión médica, UD4. Sistemas de telemedicina y E-salud y UD5. Dispositivos de telemonitorización y servicios de E-salud), la estrategia de aprendizaje activo propuesta se aplica desde el curso 2018-19 al finalizar la "UD1. Organización de sistemas de información de salud", y su evaluación reemplaza el método basado en test que se aplicaba hasta entonces.

\section{Desarrollo de la innovación}

La presente innovación parte del análisis y alineamiento con los objetivos pedagógicos establecidos para la materia, en forma de resultados de aprendizaje y competencias transversales, descritos en la guía docente de la asignatura (GUIA DOCENTE SIT1, 2018). Relacionados con la UD1, objeto de esta propuesta, podemos destacar los resultados mostrados en la Tabla 1, los cuales estarían alienados con el diseño y gestión de un sistema de información hospitalaria, y el soporte informático a las actividades de salud y gestión desarolladas en todos los servicios de un hospital. En cuanto a las competencias transversales, si bien actualmente la guía docente incluye las competencias "(CT-11) Aprendizaje permanente" y "(CT-13) Instrumental específica", gestionadas principalmente por el trabajo académico evaluable de la asignatura, esta propuesta de aprendizaje basado en juego de roles premite además trabajar nuevas competencias como "(CT-04) Innovación, creatividad y emprendimiento", "(CT-06) Trabajo en equipo y liderazgo", "(CT-08) Comunicación efectiva”, y “(CT-09) Pensamiento crítico”.

Tabla 1. Selección de resultados de aprendizaje de la asignatura "Sistemas de Información y Telemedicina 1" alineados con lainnovación propuesta

\section{Código competencia}

$10(\mathrm{ES})$

\section{Resultado de aprendizaje}

Tener conocimientos de los fundamentos científico-tecnológicos en los que se basa la tecnología médica en diferentes escalas: macro, micro y nano.

9(ES) Capacidad de identificar, formular y resolver problemas en la interfaz entre la tecnología y las ciencias de la salud, biología y medicina.

16(ES) Capacidad de proyectar, diseñar, desarrollar, instalar, utilizar y mantener procedimientos, dispositivos, equipos y sistemas para la prevención, diagnostico, tratamiento y rehabilitación.

$5(\mathrm{ES})$

Poseer conocimientos de herramientas informáticas para analizar, calcular, visualizar, representar y obtener la información necesaria para apoyar las tareas de análisis, cálculo, diseño, desarrollo y gestión relacionadas con la ingeniería biomédica.

$11(\mathrm{ES})$

Ser capaz de entender las características técnicas y funcionales de los sistemas, métodos y procedimientos que se utilizan en prevención, diagnóstico, terapia y rehabilitación.

Por otra parte, es importante considerar en el diseño la configuración del alumnado y asignatura. Se trata de una asignatura de $4^{\circ}$ curso, donde los alumnos ya disponen de las bases fundamentales de conocimiento en su disciplina de ingeniería biomédica. Al tratarse de la primera unidad temática de la asignatura, los alumnos comienzan a familizarizarse con los conceptos específicos de sistemas de 
información y análisis de datos biomédicos, si bien la actividad está planificada al final de dicha unidad. Las sesiones teóricas de la asignatura disponen de 2 horas. Finalmente, contamos con un número de aproximadamente 60 alumnos.

A continuación, describimos los apartados que conforman el diseño y evaluación de la innovación propuesta.

\subsection{Guión de la actividad}

La actividad está dividida en una parte presencial y otra no presencial. La presencial está diseñada con una duración de 2 horas, y contempla la explicación y desarrollo del juego de roles en el aula por grupos, así como la recolección de retroalimentación de la misma. La parte no presencial incluye la lectura previa del contenido teórico del último bloque de la Unidad 1 en la que se enmarca la actividad y, tras el desarrollo de la parte presencial, el terminar de completar y revisar entre todo el grupo el entregable final que se evaluará.

A fin de cubrir los distintos resultados de aprendizaje de la unidad, decidimos diseñar el juego de roles de forma que los alumnos, representando a jefes de los distintos servicios de un hospital y compañías de software, negocian la adquisición de un nuevo componente de sistemas de información en función de las necesidades de su departamento y globales al hospital. Estos componentes de sistemas de información para los distintos servicios hospitalarios, así su entorno tecnológico, han sido estudiados previamente en la unidad.

A continuación mostramos el guión del juego de roles tal y como se les proporciona a los alumnos:

(Duración total: 1,5h, grupos de 8 alumnos, los alumnos habrán leído previamente el Tema 1.3)

El hospital dispone de una partida económica para la adquisición de un nuevo componente de sistemas de información hospitalarios que mejore alguno de los existentes. Actualmente se está llevando a cabo un proceso de negociación entre el equipo de dirección del hospital a fin de llegar a una decisión. Cada miembro del equipo de dirección defiende las necesidades del servicio del que es responsable. En la negociación participan dos representantes de las empresas $A$ y $B$ que ofrecen distintas aproximaciones para el componente a adquirir.

El objetivo de la actividad es decidir qué componente adquirir y de qué tipo de los ofrecidos por las empresas A y B. El componente será uno de los descritos en el Tema 1.3, si bien puede definirse uno que por necesidad de funcionamiento componga como máximo dos de ellos. La solución puede incluir software, hardware o ambos.

Los roles a representar por los alumnos están recogidos en la Tabla adjunta. (Tabla 2 en este trabajo)

Al comienzo de la clase se explicará la actividad y seguidamente se entregará un sobre a cada grupo con las descripciones de los roles para que se extraigan y asignen a los distintos miembros del grupo de forma aleatoria (15 minutos). Seguidamente, se dispondrá de 30 minutos para que cada alumno prepare su rol con el material de la Unidad 1, internet y con la ayuda del profesor. La negociación durará 45 minutos. Posteriormente, se acordará y escribirá el acta durante 15-30 minutos. Al finalizar la actividad se pasará un cuestionario online en el que cada alumno introducirá que rol ha interpretado, su retroalimentación sobre el aprendizaje mediante la actividad, y el grado de satisfacción.

Evaluación mediante acta de la reunión máximo 2 páginas. 
El acta debe:

- Incluir las opiniones de todos los roles.

- Justificar las decisiones tomadas con criterios objetivos.

- Demostrar conocimiento de los aspectos más importantes tratados a lo largo del Tema $1(1.1,1.2$ y 1.3$)$.

La Tabla 2 muestra los roles propuestos para la actividad así como un guión orientativo para cada uno de ellos. Se pretende una interacción directa entre todos los roles, si bien existen algunos de ellos que tienen papeles soporte a la actividad, concretamente el Gerente de hospital, Representante de los secretarios/as médicos, y las dos empresas. Es decir, en caso de que no se tengan 8 miembros en un grupo (por el número de alumnos disponibles), éstos roles no pueden eliminarse, sí en cambio los restantes, que defienden sus distintos departamentos (aunque es recomendable también mantener el Director/a de sistemas de información, por las propias características de la asignatura y actividad).

Tabla 2. Roles y guión orientativo para cada uno de ellos

\begin{tabular}{|c|c|}
\hline Rol & Guión orientativo \\
\hline Gerente hospital & $\begin{array}{l}\text { Quiere un subsistema que le ahorre costes, por ejemplo, para asistencia remota } \\
\text { de pacientes, soporte a la toma de decisiones, cuadro de mandos, etc. }\end{array}$ \\
\hline $\begin{array}{l}\text { Director/a sistemas de } \\
\text { información }\end{array}$ & $\begin{array}{l}\text { Extremadamente concienciado con la seguridad. Le gustaría disponer de } \\
\text { herramientas que mejoren el trabajo de interconectar diferentes subsistemas. }\end{array}$ \\
\hline Director/a servicio radiología & $\begin{array}{l}\text { Preocupado porque el hospital comienza a tener problemas de almacenamiento } \\
\text { y procesamiento de datos de imagen y por la baja eficiencia del proceso de } \\
\text { crear informes radiológicos manualmente en el sistema RIS. }\end{array}$ \\
\hline Director/a servicio laboratorio & $\begin{array}{c}\text { El laboratorio funciona prácticamente siempre en niveles de saturación. } \\
\text { Protesta porque se piden más pruebas de las necesarias, y defiende la necesidad } \\
\text { de un sistema que optimice el número de pruebas solicitadas. Además, la } \\
\text { incorporación de análisis genómicos al laboratorio está empezando a generar } \\
\text { problemas de almacenamiento. }\end{array}$ \\
\hline Director/a médico & $\begin{array}{c}\text { Lleva años protestando por un registro médico electrónico (EMR) arcaico, que } \\
\text { da lugar en ocasiones incluso a errores médicos, debido a interfaces gráficas } \\
\text { pobres y a elevados tiempos de espera. Cuenta con el apoyo del director/a } \\
\text { enfermería. }\end{array}$ \\
\hline $\begin{array}{l}\text { Representante de secretarios/as } \\
\text { médicos }\end{array}$ & $\begin{array}{l}\text { Desearía un sistema que ahorre trabajo al procesar los textos médicos del } \\
\text { EMR, tanto del texto libre como la corrección de datos de pacientes erróneos. } \\
\text { Anota los detalles de la reunión y apacigua las discusiones. }\end{array}$ \\
\hline Representante empresa A & $\begin{array}{l}\text { Ofrece soluciones basadas en Cloud Computing y/o en Sistemas de } \\
\text { Información Médica altamente probados aunque cerrados. }\end{array}$ \\
\hline Representante empresa B & $\begin{array}{l}\text { Ofrece soluciones basadas en Open Source sin salir del hospital y/o en } \\
\text { Sistemas de Información Médica a medida. }\end{array}$ \\
\hline
\end{tabular}

$\mathrm{Al}$ inicio de la actividad y tras explicar el guión, se recomienda que inicialmente cada miembro del hospital exponga sus necesidades, posteriormente las empresas propongan qué ofrecen según sus características para resolver los problemas planteados, y por último se negocia entre todos los participantes qué componente adquirir, o bien inlcuso combinar varios de ellos con un soporte tecnológico común. Actualmente esta organización es voluntaria, y cada grupo puede organizar la negociación como decidan. Si bien podría ser interesante como trabajo futuro estudiar una organización común para todos. Durante toda la negociación, el profesor puede servir de apoyo ante cualquier duda o problema.

Como podemos observar, el guión ofrecido a los alumnos describe los principales aspectos de la evaluación de la actividad. Ésta se realiza actualmente a partir de un acta de la reunión, tal y como se 
escribiría en dicha actividad en la realidad, donde se exponen los puntos aportados por cada participante así como las conclusiones. Se otorga la responsabilidad de escritura de actua durante la reunión al Representante de los secretarios/as médicos, si bien este borrardor puede ser revisado y completado por el resto de miembros del grupo durante una semana más hasta su entrega. La rúbrica para la evaluación de la misma se describe en la sección siguiente.

\subsection{Rúbrica de evaluación}

Con el fin de definir una rúbrica para la evaluación de la actividad, inicialmente se realizó una búsqueda online sobre rúbricas ya definidas para la evaluación de actividades de juego de roles, con la cual poder comparar nuestra propuesta inicial y llegar a un consenso final. Encontramos un total de 6 rúbricas, las cuales cubrían distintos aspectos y resultados de aprendizaje. En la Tabla 3, hemos identificado y alineado por equivalencia los principales aspectos a evaluar de las distintas rúbricas. En la última columna incluimos nuestra propuesta. Las rúbricas encontradas están enmarcadas en juegos de roles para distintos ámbitos, por ejemplo el trabajo de Gaete-Quezada representa entrevistas de trabajo, mientras que el de Joyner and Young actos médicos. El resto resultaron más bien genéricas. Por otra parte, algunos trabajos incluían el detalle de cada celda, mientras que la mayoría de ellos únicamente indicaba la puntuación. Finalmente, todos los trabajos consideraban la evaluación de la propia representación, algo que actualmente no hemos considerado en nuestra propuesta, pero cuya evaluación equivalente hemos enlazado con otros conceptos. Cabe decir que esta comparación no puede resultar exhaustiva, dadas las diferencias comentadas, por este motivo varios elementos han sido replicados en distintas filas, ya que podrían considerarse parte de distintas evaluaciones conforme a nuestros objetivos. En cualquier caso, esta comparación ha ayudado para agrupar nuestra propuesta y redactar los propios umbrales de evaluación para cada celda en la rúbrica definitiva, la cual recogemos en la Tabla 4.

Tabla 3. Aspectos a evaluar de acuerdo a distintas rúbricas empleadas en la literatura para juegos de roles, hacia el desarrollo de la propuesta

\begin{tabular}{|c|c|c|c|c|c|c|}
\hline $\begin{array}{l}\text { (Gaete- } \\
\text { Quezada, } \\
\text { 2011) }\end{array}$ & $\begin{array}{c}\text { (ROLE-PLAY } \\
\text { RUBRIC BLM } \\
\text { G-17) }\end{array}$ & $\begin{array}{l}\text { (iRUBRIC - } \\
\text { LXW53A9) }\end{array}$ & $\begin{array}{l}\text { (Joyner and } \\
\text { Young, 2006) }\end{array}$ & $\begin{array}{c}\text { (RUBISTA } \\
\text { R) }\end{array}$ & $\begin{array}{c}\text { (SCRIBD - } \\
\text { MO110 } \\
\text { SPRING } \\
\text { 2015) }\end{array}$ & $\begin{array}{l}\text { Propuesta } \\
\text { actual }\end{array}$ \\
\hline $\begin{array}{l}\text { Guión / Tipo } \\
\text { de entrevista }\end{array}$ & $\begin{array}{l}\text { Role play was well } \\
\text { prepared and } \\
\text { organized / Role } \\
\text { played in a } \\
\text { convicent, } \\
\text { consistent manner }\end{array}$ & Organization & $\begin{array}{l}\text { Management } \\
\text { plan }\end{array}$ & Preparation & - & $\begin{array}{l}\text { Justificación } \\
\text { decisión / } \\
\text { Presentación }\end{array}$ \\
\hline $\begin{array}{l}\text { Aplicación de } \\
\text { teorías }\end{array}$ & - & $\begin{array}{l}\text { Quality of } \\
\text { content }\end{array}$ & Clinical skills & Content & $\begin{array}{c}\text { List of } \\
\text { resources / } \\
\text { Medical } \\
\text { terms }\end{array}$ & Contenido tema \\
\hline $\begin{array}{c}\text { Participación } \\
\text { grupo }\end{array}$ & $\begin{array}{c}\text { Arguments and } \\
\text { viewpoints } \\
\text { expressed fit }\end{array}$ & $\begin{array}{l}\text { Presentation } \\
\text { skills }\end{array}$ & - & Roles & Role play & $\begin{array}{l}\text { Representación } \\
\text { roles }\end{array}$ \\
\hline- & Audience attraction & Impact & $\begin{array}{c}\text { Communicatio } \\
n \text { skills }\end{array}$ & $\begin{array}{c}\text { Overall } \\
\text { impression }\end{array}$ & Script / Notes & Presentación \\
\hline $\begin{array}{l}\text { Varios aspectos } \\
\text { relacionados } \\
\text { con el dominio } \\
\text { de las } \\
\text { entrevistas }\end{array}$ & $\begin{array}{l}\text { Audience attraction } \\
\text { / Clear speech }\end{array}$ & $\begin{array}{c}\text { Presentation } \\
\text { skills }\end{array}$ & $\begin{array}{c}\text { Verbal and } \\
\text { non-verbal } \\
\text { communication }\end{array}$ & $\begin{array}{c}\text { Overall } \\
\text { impression }\end{array}$ & $\begin{array}{c}\text { Communicati } \\
\text { on skills }\end{array}$ & $\begin{array}{l}\text { Parcialmente } \\
\text { evaluado en } \\
\text { Presentación }\end{array}$ \\
\hline
\end{tabular}


Tabla 4. Rúbrica de evaluación propuesta

\begin{tabular}{|c|c|c|c|c|c|}
\hline & 10 & 7,5 & 5 & 2,5 & 0 \\
\hline 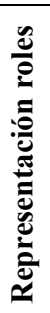 & $\begin{array}{l}\text { Todos los roles se } \\
\text { ajustan a lo descrito } \\
\text { en el guión y todos } \\
\text { sus argumentos son } \\
\text { adecuados }\end{array}$ & $\begin{array}{l}\text { En general, los roles } \\
\text { se ajustan a lo } \\
\text { descrito en el guión } \\
\text { y algunos } \\
\text { argumentos son } \\
\text { adecuados }\end{array}$ & $\begin{array}{l}\text { Algunos roles se } \\
\text { ajustan a lo } \\
\text { descrito en el } \\
\text { guión y algunos } \\
\text { argumentos son } \\
\text { adecuados }\end{array}$ & $\begin{array}{l}\text { Algunos roles se } \\
\text { ajustan a lo } \\
\text { descrito en el } \\
\text { guión, pero los } \\
\text { argumentos no } \\
\text { son adecuados }\end{array}$ & $\begin{array}{c}\text { La } \\
\text { representación } \\
\text { de los roles no } \\
\text { se ajusta a lo } \\
\text { descrito en el } \\
\text { guión }\end{array}$ \\
\hline 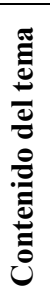 & $\begin{array}{c}\text { Todos los roles } \\
\text { emplean de forma } \\
\text { consistente aspectos } \\
\text { teóricos y vocabulario } \\
\text { del tema, y se } \\
\text { demuestra estudio } \\
\text { adicional }\end{array}$ & $\begin{array}{l}\text { En general, los roles } \\
\text { emplean de forma } \\
\text { consistente aspectos } \\
\text { teóricos y } \\
\text { vocabulario del tema }\end{array}$ & $\begin{array}{c}\text { Se emplean de } \\
\text { forma general } \\
\text { contenido del tema } \\
\text { si bien la } \\
\text { consistencia es } \\
\text { mejorable }\end{array}$ & $\begin{array}{c}\text { Algun contenido } \\
\text { teórico es } \\
\text { expuesto por } \\
\text { parte de los roles }\end{array}$ & $\begin{array}{l}\text { No se demuestra } \\
\text { haber tratado } \\
\text { ningún } \\
\text { contenido } \\
\text { teórico del tema }\end{array}$ \\
\hline 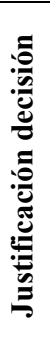 & $\begin{array}{l}\text { Se demuestra una } \\
\text { profunda } \\
\text { comprensión y } \\
\text { análisis al haber } \\
\text { tomado la decisión } \\
\text { escogida, conforme a } \\
\text { los roles e interacción } \\
\text { entre ellos }\end{array}$ & $\begin{array}{c}\text { La decisión escogida } \\
\text { es adecuada } \\
\text { conforme a los roles } \\
\text { y se muestra } \\
\text { interacción }\end{array}$ & $\begin{array}{c}\text { La decisión } \\
\text { escogida es } \\
\text { adecuada y se } \\
\text { alinéa con algun } \\
\text { rol, pero sin } \\
\text { mostrar } \\
\text { interacción entre } \\
\text { ellos }\end{array}$ & $\begin{array}{c}\text { Se toma una } \\
\text { decisión } \\
\text { adecuada pero } \\
\text { no se justifica } \\
\text { bien conforme a } \\
\text { los roles }\end{array}$ & $\begin{array}{c}\text { La decisión final } \\
\text { parece haber } \\
\text { sido tomada de } \\
\text { forma arbitraria }\end{array}$ \\
\hline & $\begin{array}{c}\text { Se ajusta a las } 2 \\
\text { páginas, organiza } \\
\text { claramente exposición } \\
\text { roles y justificación } \\
\text { decisión, buena } \\
\text { legibilidad e } \\
\text { impresión general }\end{array}$ & $\begin{array}{c}\text { Se ajusta a las } 2 \\
\text { páginas, organiza } \\
\text { claramente } \\
\text { exposición roles y } \\
\text { justificación } \\
\text { decisión, pero la } \\
\text { legibilidad e } \\
\text { impresión general es } \\
\text { mejorable }\end{array}$ & $\begin{array}{l}\text { Se ajusta a las } 2 \\
\text { páginas, pero la } \\
\text { organización del } \\
\text { contenido no es } \\
\text { adecuada }\end{array}$ & $\begin{array}{c}\text { No se ajusta a } \\
\text { las } 2 \text { páginas a } \\
\text { pesar de tener } \\
\text { una organización } \\
\text { adecuada }\end{array}$ & $\begin{array}{l}\text { No se ajusta a } \\
\text { las } 2 \text { páginas y } \\
\text { la organización } \\
\text { no es adecuada }\end{array}$ \\
\hline
\end{tabular}

\subsection{Cuestionario de retroalimentación}

A fin de evaluar el aprendizaje percibido por los alumnos y obtener su retroalimentación para la mejora continua de la actividad, la última parte presencial de la actividad consiste en recoger una serie de respuestas a cuestionarios. Para la recogida de estas respuestas se emplea la herramienta Socrative ${ }^{1}$, accediendo los alumnos en tiempo real desde sus teléfonos móviles de forma anónima. Las preguntas iniciales han sido inspiradas en las preguntas de retroalimentación que ofrece la herramienta Kahoot! ${ }^{2}$ tras finalizar una actividad, herramienta que empleamos para reforzar el aprendizaje tras cada clase. El cuestionario consiste en las siguientes preguntas, cuya respuesta en cada caso es opcional:

- Q1. ¿Cómo de divertido ha sido? [Muy divertido, Divertido, Neutral, Poco, Muy poco]

- Q2. ¿Has aprendido algo? [Sí, No]

\footnotetext{
${ }^{1}$ https://socrative.com/

${ }^{2}$ https://kahoot.com/
} 
- Q3. ¿Lo recomendarías? [Sí, No]

- Q4. ¿Cómo te sientes? [Positivo, Neutral, Negativo]

- Q5. ¿Cuál ha sido tu rol? [selección del conjunto de roles de la actividad]

- Q6 .Comentarios o sugerencias [respuesta de texto libre]

En la siguiente sección mostramos los resultados de estos cuestionarios de retroalimentación en los cursos en que la actividad ha sido llevada a cabo hasta el momento.

\section{Resultados}

El juego de roles propuesto en este trabajo ha sido llevado a la práctica en los últimos dos cursos, 2018-19 y 2019-20. En el curso 2018-19 el número de alumnos participantes fue de 60 y en el 2019-20 de 64. Se realizaron 8 grupos en ambos cursos, debiendo limitar en algunos de ellos el número de roles a 7 en lugar de los 8 totales, lo que no impide el desarrollo de la actividad manteniendo los roles fundamentales, como describimos anteriormente.

Las Tablas 5, 67 y 8 muestran los resultados de las preguntas Q1 a Q4, respectivamente. De acuerdo a los resultados de Socrative, se recibieron 70 y 52 cuestionarios, lo que evidencia un posible problema en el curso 2018-19, si bien el número de preguntas no respondidas puede evidenciar problemas de conexión y reconexiones, lo que no hemos considerado significativo para descartar el resto de respuestas.

En cuanto a los resultados, podemos observar que en su gran mayoría las respuestas son positivas. En la pregunta Q1. ¿Cómo de divertido ha sido? Los alumnos han mostrado su satisfacción positiva en cuanto al entretenimiento obtenido durante la actividad. Destacamos especialmente el elevado porcentaje positivo en la pregunta Q2. ¿Has aprendido algo?., del 87,1\% y 96,2\% en sendos cursos.

Tabla 5. Respuestas a pregunta Q1. ¿Cómo de divertido ha sido?

\begin{tabular}{ccccccc}
\hline Curso / Respuesta & $\begin{array}{c}\text { Muy } \\
\text { divertido }\end{array}$ & Divertido & Neutral & Poco & Muy poco & $\begin{array}{c}\text { Sin } \\
\text { respuesta }\end{array}$ \\
\hline $2018-19$ & $10(14,2 \%)$ & $32(45,7 \%)$ & $16(22,9 \%)$ & $4(5,7 \%)$ & $1(1,4 \%)$ & $7(10 \%)$ \\
\hline $2019-20$ & $11(21,2 \%)$ & $28(53,8 \%)$ & $12(23,1 \%)$ & $0(0 \%)$ & $0(0 \%)$ & $1(1,9 \%)$ \\
\hline
\end{tabular}

Tabla 6. Respuestas a pregunta Q2. ¿Has aprendido algo?

\begin{tabular}{cccc}
\hline Curso/ Respuesta & Sí & No & $\begin{array}{c}\text { Sin } \\
\text { respuesta }\end{array}$ \\
\hline $2018-19$ & $61(87,1 \%)$ & $3(4,3 \%)$ & $6(8,6 \%)$ \\
\hline $2019-20$ & $50(96,2 \%)$ & $1(1,9 \%)$ & $1(1,9 \%)$ \\
\hline
\end{tabular}

Tabla 7. Respuestas a pregunta Q3. ¿Lo recomendarias?

\begin{tabular}{cccc}
\hline Curso / Respuesta & Sí & No & $\begin{array}{c}\text { Sin } \\
\text { respuesta }\end{array}$ \\
\hline $2018-19$ & $47(67,1 \%)$ & $10(14,2 \%)$ & $\begin{array}{c}13 \\
(18,6 \%)\end{array}$ \\
\hline $2019-20$ & $48(92,3 \%)$ & $4(7,7 \%)$ & $0(0 \%)$ \\
\hline
\end{tabular}


Tabla 8. Respuestas a pregunta Q4. ¿Cómo te sientes?

\begin{tabular}{ccccc}
\hline Curso / Respuesta & Positivo & Neutral & Negativo & $\begin{array}{c}\text { Sin } \\
\text { respuesta }\end{array}$ \\
\hline $2018-19$ & $30(42,6 \%)$ & $29(41,4 \%)$ & $6(8,6 \%)$ & $5(7,1 \%)$ \\
\hline $2019-20$ & $34(65,4 \%)$ & $17(32,7 \%)$ & $0(0 \%)$ & $1(1,9 \%)$ \\
\hline
\end{tabular}

Respecto a las preguntas de comentarios y sugerencias, se recibió una elevada cantidad de respuestas, tanto de comentarios positivos como de sugerencias constructivas para mejorar. En la Tabla 9 mostramos una selección de dichas respuestas, agrupadas en los siguientes ámbitos de aplicación: organización, roles, evaluación y otras. Adicionalmente, hemos resumido con nuestras palabras los casos en que habían múltiples comentarios equivalentes, representado con texto sin entrecomillar, y también mantenido respuestas escritas directamente por los alumnos con texto entrecomillado.

En cuanto a la organización, encontramos que en ambos cursos, los alumnos sugieren una mayor explicación previa tanto de la actividad como de la unidad temática originalmente propuesta para su estudio previo en casa. También se propone el realizar la explicación y asignación de grupos y roles la clase anterior, a fin de preparar en casa la misma, y realizar la actividad posteriormente en clase. Algunos alumnos indican falta de tiempo, sin embargo otros sugieren terminar por completo la actividad en clase.

En cuanto a los roles, de acuerdo a las opiniones de los alumnos hemos encontrado un cuello de botella en los roles correspondientes a las empresas que ofrecen los componentes software, detectando una necesidad de mayor explicación sobre ellos. También se ha mencionado la gratitud por ponerse en el punto de vista de los roles.

En cuanto a la evaluación, se ha propuesto el hacer presentaciones delante de la clase por parte de cada grupo, con intercambio de opiniones entre los grupos, así como no realizar memoria y evaluar la propia presentación.

Finalmente, sobre otros comentarios, se enviaron mensajes expresando la satisfacción con la actividad, destacando la dinámica y trabajo en equipo como método de aprendizaje activo. También se propuso realizar la actividad en inglés o valenciano. 
Tabla 9. Respuestas a comentarios o sugerencias, agrupadas por ámbito de aplicación, entrecomilladas las respuestas literales, sin entrecomillar resumiendo varias respuestas similares

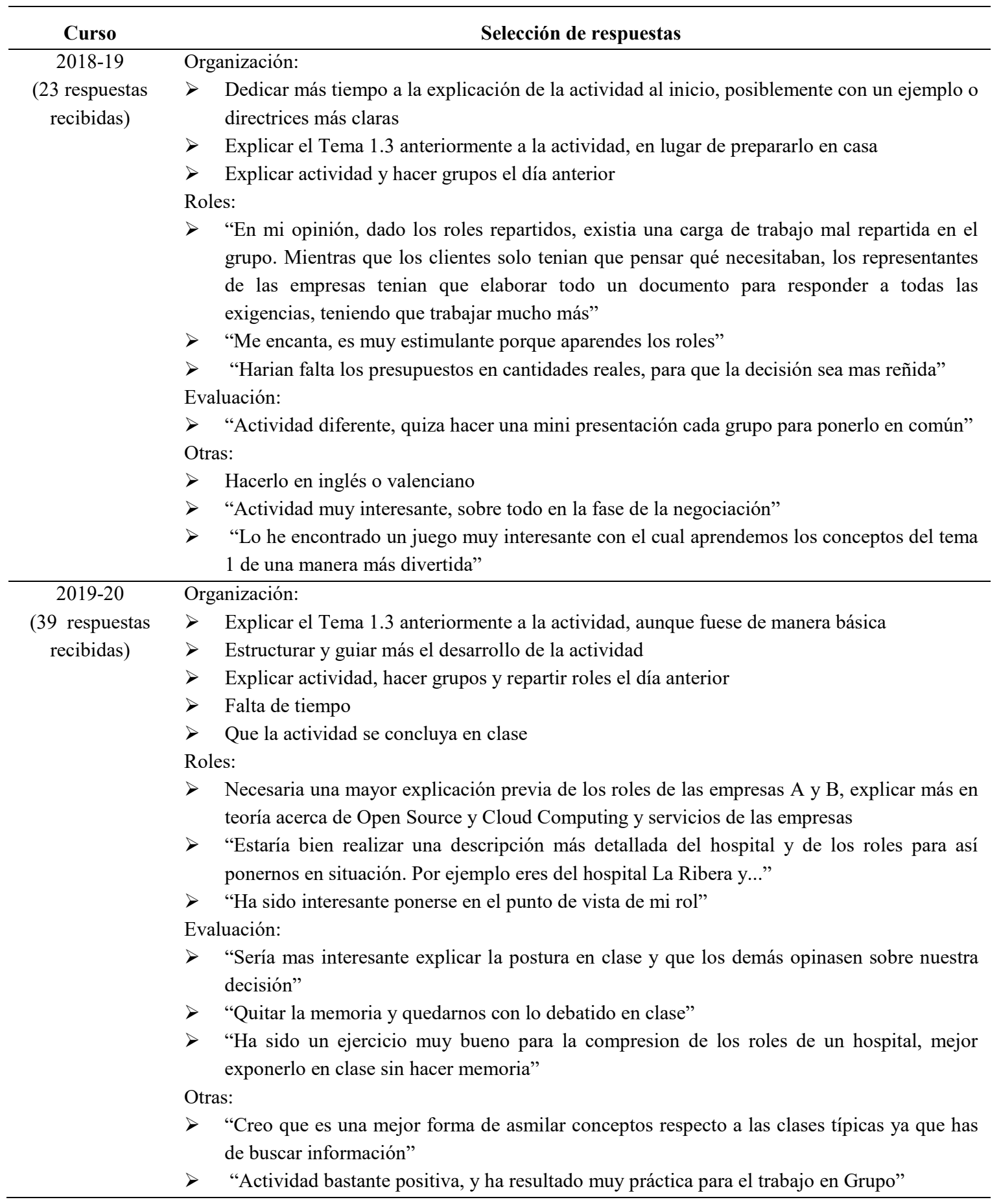

\section{Conclusiones}

Hemos propuesto una nueva actividad de aprendizaje activo mediante juego de roles en el Grado en Ingeniería Biomédica de la UPV, en la que los alumnos de Sistemas de Información y Telemedina aprenden de forma activa parte de los resultados de aprendizaje de la asignatura, y trabajan al mismo tiempo nuevas competencias transverales. La actividad simula la negociación de la adquisición de un 
sistema de información hospitalaria, y los alumnos representan roles de distintos jefes de servicio del hospital y empresas desarrolladoras de software para dichos componentes.

Aplicada desde el curso 2018-19, la actividad ha demostrado su efectividad, cumpliendo los objetivos pedagógicos establecidos para la materia, con una buena apreciación del aprendizaje recibido por los alumnos, y presentando una elevada tasa de retroalimentación.

Para mejorar la actividad en los próximos cursos, gracias a la retroalimentación proporcionada por los alumnos, consideraremos los siguientes aspectos. Proporcionaremos una explicación previa de la actividad y de la última sub-unidad necesaria para su desarrollo, si bien mantendremos trabajo preparatorio en casa. Reforzaremos la explicación de los roles de las empresas proveedoras de software. Ofreceremos un ejemplo más claro de como organizar el proceso de la negociación. Por último, valoraremos la viabilidad, en función del tiempo disponible, de desarrollar y evaluar una representación en el aula en lugar de la memoria del acta de la reunión.

\section{Agradecimientos}

Queremos dar las gracias a todos los alumnos de Sistemas de Información y Telemedicina I de los cursos 2018-19 y 2019-20 por su gran motivación en el desarrollo del juego de roles y por su demostración de interés en mejorar la actividad en futuros cursos gracias a los comentarios y sugerencias aportadas. , de los cuales también hemos aprendido nosotros.

\section{Referencias}

GAETE-QUEZADA, R.A. (2011). "El juego de roles como estrategia de evaluación de aprendizajes universitarios". Educ.Educ. Vol. 14, No. 2, 289-307.

GUIA DOCENTE SIT1 (2018). Asignatura "Sistemas de Información y Telemedicina 1" (13068), Grado en Ingeniería Biomédica (175), E.T.S.I. Industriales, Universitat Politècnica de València.

IRUBRIC - LXW53A9: TEAM ROLE-PLAY PRESENTATION ASSESSMENT RUBRIC. https://www.rcampus.com/rubricshowc.cfm?code=LXW53A9\&sp=yes. [Consulta: 23 de marzo 2020].

JOYNER, B. y YOUNG, L. (2006). "Teaching medical students using role play: Twelve tips for successful role plays”. Medical Teacher, Vol. 28, No. 3, 225-229.

McCONVILLE, J., RAUCH, S., HELGEGREN, I. y KAIN, J. (2017), "Using role-playing games to broaden engineering education". International Journal of Sustainability in Higher Education, Vol. 18 No. 4, pp. 594-607.

PONSA, P., VILANOVA, R., y AMANTE, B. (2010). "The use of role playing in engineering curricula: a case study in human-automation systems". En: IEEE EDUCON 2010 Conference. 1335-1341.

ROLE-PLAY RUBRIC BLM G-17. https://www.edu.gov.mb.ca/k12/cur/socstud/frame_found_sr2/g_blms/g-17.pdf. [Consulta: 23 de marzo 2020].

RUBISTAR. Role-Playing Rubric. Advanced Learning Technologies in Education Consortia ALTEC. http://rubistar.4teachers.org/index.php?screen=PrintRubric\&rubric_id=1175361. [Consulta: 23 de marzo 2020].

SCRIBD - MO110 SPRING 2015 by Merboy Pangilinan. Role Play \& Case Study Rubric. https://www.scribd.com/document/431940156/ROLE-PLAYING-Rubric-copy-docx. [Consulta: 23 de marzo 2020].

SIMPSON, M.A. (1985). “How to Use Role-Play in Medical Teaching”. Medical Teacher, Vol. 7, No. I.

STEINERT, Y. (1993). "Twelve tips for using role-plays in clinical teaching”. Medical Teacher, 15:4, 283-291.

SVENSSON, R.B. y REGNELL, B. (2017). "Is role playing in Requirements Engineering Education increasing learning outcome?”. Requirements Eng. 22, 475-489. 